\title{
SPATIAL RESOLUTION AND NOISE PROPERTIES OF REGULARIZED MOTION-COMPENSATED IMAGE RECONSTRUCTION
}

\author{
Se Young Chun and Jeffrey A. Fessler \\ Department of Electrical Engineering and Computer Science \\ The University of Michigan \\ 1301 Beal Avenue, Ann Arbor, MI 48109-2122
}

\begin{abstract}
Reducing motion artifacts is an important problem in medical image reconstruction. Using gating to partition data into separate frames can reduce motion artifacts but can increase noise in images reconstructed from individual frames. One can pool the frames to reduce noise by using motion-compensated image reconstruction (MCIR) methods.

MCIR methods have been studied in many medical imaging modalities to reduce both noise and motion artifacts. However, there has been less analysis of the spatial resolution and noise properties of MCIR methods. This paper analyzes the spatial resolution and noise properties of MCIR methods based on a general parametric motion model. For simplicity we consider the motion to be given. We present a method to choose quadratic spatial regularization parameters to provide predictable resolution properties that are independent of the object and the motion. The noise analysis shows that the estimator variance depends on both the measurement covariance and the Jacobian determinant values of the motion. A 2D PET simulation demonstrates the theoretical results.
\end{abstract}

Index Terms - motion-compensated image reconstruction, spatial resolution and noise properties, quadratic regularization parameter, space-invariant tomographs.

\section{INTRODUCTION}

Medical imaging modalities such as PET, SPECT, CT and MRI provide useful patient image information for diagnosis, treatment planning and intervention in clinical settings. However, due to their innate acquisition time limitations, there are trade-offs between image noise (SNR) and motion artifacts. Gating methods based on breathing signals or ECG signals can reduce motion artifacts, but they also reduce SNR by discarding potentially useful data.

Motion-compensated image reconstruction (MCIR) methods attempt to use all collected data and motion information at the same time to improve image quality. Many such methods use nonrigid motion models for human anatomy. Motion

\footnotetext{
This work was supported in part by NIH/NCI grant 1P01 CA87634.
}

information can be estimated separately [1-3], or simultaneously [4-6]. Often such methods can improve image quality.

However, there has been less research on spatial resolution and noise properties of MCIR methods [7]. It is important to know the statistical properties of an image reconstruction method. Mean and covariance properties have been well studied for static image reconstruction $[8,9]$ and dynamic image reconstruction [10]. Based on such analyses, regularization designs that provide spatial resolution uniformity have been proposed for quadratic regularizers [11] and nonquadratic regularizers [12].

This paper studies the spatial resolution and noise properties of MCIR methods $[1,2,4,6]$. It also proposes a strategy to determine regularization parameters that provide resolution uniformity independent of the object and motion (Section 3). Lastly, it shows that the noise properties depend on both the measurement covariance and Jacobian determinant values of the motion (Section 4). A 2D PET simulation illustrates the theory (Section 5).

\section{METHOD}

\subsection{Measurement model}

Let $t_{m}$ denote the time of the $m$ th "scan," i.e., the $m$ th frame in a gated study. We assume that the measurements are related to the object $\boldsymbol{f}_{m}=\left\{f\left(\cdot, t_{m}\right)\right\}$ linearly as follows:

$$
\boldsymbol{y}_{m}=\boldsymbol{G}_{m} \boldsymbol{f}_{m}+\boldsymbol{\epsilon}_{m}
$$

where $\boldsymbol{y}_{m}$ denotes the measurements for the $m$ th frame, $\boldsymbol{G}_{m}$ denotes the system model for the $m$ th frame and $\boldsymbol{\epsilon}_{m}$ denotes noise. We assume that the object $\boldsymbol{f}_{m}$ and measurement $\boldsymbol{y}_{m}$ are motion-free, i.e., the object does not move during the $m$ th scan (gate or frame). We allow the system model to possibly differ for each frame to accommodate systems that rotate such as gated SPECT or CT or that can otherwise change sampling properties dynamically such as MRI. 


\subsection{Motion-compensated image reconstruction model}

For MCIR methods, we first need to choose a reference image frame among $\left\{\boldsymbol{f}_{1}, \cdots, \boldsymbol{f}_{M}\right\}$. Without loss of generality, we assume that $\boldsymbol{f}_{1}$ is our reference image frame. Then, the rest of the image frames are represented as a warped version of $f_{1}(\underline{r})$ :

$$
\boldsymbol{f}_{m}=\boldsymbol{T}_{m, 1} \boldsymbol{f}_{1}, \quad m=1, \ldots, M,
$$

where $\boldsymbol{T}_{m, 1}$ is an image warp that maps $f_{1}(\underline{r})$ to $f_{m}(\underline{r})$.

Substituting (2) into (1) yields a measurement model that depends only on one image $\boldsymbol{f}_{1}$ instead of all images $\boldsymbol{f}_{m}$ :

$$
\boldsymbol{y}_{m}=\boldsymbol{G}_{m} \boldsymbol{T}_{m, 1} \boldsymbol{f}_{1}+\boldsymbol{\epsilon}_{m}, \quad m=1, \ldots, M
$$

Stacking up these models yields the overall model

$$
\boldsymbol{y}_{\mathrm{c}}=\boldsymbol{G}_{\mathrm{d}} \boldsymbol{T}_{\mathrm{c}} \boldsymbol{f}_{1}+\boldsymbol{\epsilon}_{\mathrm{c}},
$$

where $\boldsymbol{G}_{\mathrm{d}}=\operatorname{diag}\left\{\boldsymbol{G}_{1}, \cdots, \boldsymbol{G}_{M}\right\}, \boldsymbol{T}_{\mathrm{c}}=\left[\boldsymbol{T}_{1,1}^{\prime}, \cdots, \boldsymbol{T}_{M, 1}^{\prime}\right]^{\prime}$, and $\boldsymbol{\epsilon}_{\mathrm{c}}=\left[\boldsymbol{\epsilon}_{1}^{\prime}, \cdots, \boldsymbol{\epsilon}_{M}^{\prime}\right]^{\prime} . \boldsymbol{T}_{1,1}$ is an identity matrix. One can determine $\boldsymbol{T}_{\mathrm{c}}$ from the measurements $\boldsymbol{y}_{\mathrm{c}}$ or from other measurements, e.g., from CT in PET-CT systems. Here we treat $\boldsymbol{T}_{\mathrm{c}}$ as predetermined (known).

One can use (3) with any statistical image reconstruction objective function such as penalized likelihood. In this paper, we use a penalized weighted least square function [13]:

$$
\hat{\boldsymbol{f}}_{\mathrm{MCIR}} \triangleq \underset{\boldsymbol{f}_{1}}{\operatorname{argmin}}\left\|\boldsymbol{y}_{\mathrm{c}}-\boldsymbol{G}_{\mathrm{d}} \boldsymbol{T}_{\mathrm{c}} \boldsymbol{f}_{1}\right\|_{\boldsymbol{W}}^{2}+\eta\left\|\boldsymbol{C} \boldsymbol{f}_{1}\right\|^{2},
$$

where $\boldsymbol{W}$ is a weight matrix that approximates the inverse of the covariance of $\boldsymbol{y}_{\mathrm{c}}, \eta$ is a regularization parameter, and $C$ is a finite-difference matrix for the first or second order neighbors. Spatial resolution and noise properties also can be obtained in more general statistical formulations by similar arguments as those in [11].

\section{SPATIAL RESOLUTION PROPERTIES}

We are interested in the local impulse response of the estimator $\hat{\boldsymbol{f}}_{\mathrm{MCIR}}$. From (4) the expected value of the estimator $\hat{f}_{\mathrm{MCIR}}$ is:

$$
E\left[\hat{\boldsymbol{f}}_{\mathrm{MCIR}}\right]=\left[\boldsymbol{F}_{\mathrm{c}}+\eta \boldsymbol{R}\right]^{-1} \boldsymbol{F}_{\mathrm{c}} \boldsymbol{f}_{1},
$$

where $\boldsymbol{F}_{\mathrm{c}} \triangleq \boldsymbol{T}_{\mathrm{c}}^{\prime} \boldsymbol{G}_{\mathrm{d}}^{\prime} \boldsymbol{W} \boldsymbol{G}_{\mathrm{d}} \boldsymbol{T}_{\mathrm{c}}$ and $\boldsymbol{R} \triangleq \boldsymbol{C}^{\prime} \boldsymbol{C}$. In Poissonrelated modalities like PET and CT, the weights $\boldsymbol{W}$ depend on the object. $T_{\mathrm{c}}$ depends on the motion between image frames. Thus, (5) implies that the resolution properties of the MCIR estimator $\hat{\boldsymbol{f}}_{\text {MCIR }}$ are object and motion dependent. To our knowledge, this undesirable behavior has not been analyzed or addressed previously.

To provide uniform spatial resolution for static image reconstruction, [11] proposed a regularization design method that depends on $\boldsymbol{W}$. Here we extend this technique for MCIR methods. We first define a vector $\underline{\kappa}$ for which the following approximation holds:

$$
\boldsymbol{F}_{\mathrm{c}}=\boldsymbol{T}_{\mathrm{c}}^{\prime} \boldsymbol{G}_{\mathrm{d}}^{\prime} \boldsymbol{W} \boldsymbol{G}_{\mathrm{d}} \boldsymbol{T}_{\mathrm{c}} \approx D(\underline{\kappa}) \boldsymbol{G}_{\mathrm{d}}^{\prime} \boldsymbol{G}_{\mathrm{d}} D(\underline{\kappa}),
$$

where $D(\underline{\kappa})=\operatorname{diag}\{\underline{\kappa}\}$ is a diagonal matrix. Due to the $1 / r$ response of tomographs and the finitely supported image basis, $\boldsymbol{F}_{\mathrm{c}}$ is fairly concentrated about its diagonal. Therefore, we want $\underline{\kappa}$ to match perfectly the diagonal elements on both sides of (6) as follows:

$$
\left[\boldsymbol{T}_{\mathrm{c}}^{\prime} \boldsymbol{G}_{\mathrm{d}}^{\prime} \boldsymbol{W} \boldsymbol{G}_{\mathrm{d}} \boldsymbol{T}_{\mathrm{c}}\right]_{j j}=\kappa_{j}^{2}\left[\boldsymbol{G}_{\mathrm{d}}^{\prime} \boldsymbol{G}_{\mathrm{d}}\right]_{j j} .
$$

To further simplify, we approximate (7) as follows:

$$
\kappa_{j} \triangleq \sqrt{\frac{\left[\boldsymbol{T}_{\mathrm{c}}^{\prime} \boldsymbol{G}_{\mathrm{d}}^{\prime} \boldsymbol{W} \mathbf{1}\right]_{j}}{\left[\boldsymbol{G}_{\mathrm{d}}^{\prime} \mathbf{1}\right]_{j}}},
$$

where $\mathbf{1}=[1,1,1, \cdots]^{\prime}$. Unlike a natural extension of the conventional $\kappa$ proposed in [11] and a $\kappa$ that appeared in [7] for DFT-based covariance approximation, (8) does not con$\operatorname{tain} \boldsymbol{T}_{\mathrm{c}}^{\prime}$ in the denominator. Since one can show that

$$
\boldsymbol{T}_{m}^{\prime}=D\left(\frac{1}{\left|\nabla \boldsymbol{T}_{m}\left(\underline{r}_{j}\right)\right|}\right) \boldsymbol{T}_{m}^{-1}
$$

our proposed $\kappa$ contains additional Jacobian determinant of deformations $\left|\nabla \boldsymbol{T}_{m}\right|$. These terms compensate for the change in spatial resolution due to motion.

Extending the static case [11], we now define a modified regularizer where

$$
C \triangleq C_{0} D(\underline{\kappa}),
$$

where $C_{0}$ is the usual finite-differencing matrix. Thus, $\boldsymbol{R}=\boldsymbol{C}^{\prime} \boldsymbol{C}=D(\underline{\kappa}) \boldsymbol{C}_{0}^{\prime} \boldsymbol{C}_{0} D(\underline{\kappa})$. Substituting this regularization design into the mean expression (5) and simplifying yields

$$
E\left[\hat{\boldsymbol{f}}_{\mathrm{MCIR}}\right] \approx\left[\boldsymbol{F}_{0}+\eta \boldsymbol{C}_{0}^{\prime} \boldsymbol{C}_{0}\right]^{-1} \boldsymbol{F}_{0} \boldsymbol{f}_{1}
$$

where $\boldsymbol{F}_{0} \triangleq \sum_{m=1}^{M} \boldsymbol{G}_{m}^{\prime} \boldsymbol{G}_{m}$. Thus, our proposed regularization design (10) achieves approximately uniform spatial resolution independent of the object and motion between image frames. This aids regularization parameter selection [11] and helps match spatial resolutions of different MCIR methods. Using (10) requires additional $\boldsymbol{T}_{\mathrm{c}}^{\prime}$ computation only once at the beginning of optimization (4) and it is equivalent to the computational complexity of image interpolation for all voxels.

When $\boldsymbol{G}_{m}=\boldsymbol{G}_{0}$ for all $m$, we can match the spatial resolution of MCIR with the resolution of gated image reconstruction (gate 1). If we substitute $\eta$ with $M \eta$, then the expected value of both MCIR $\left(\hat{f}_{\mathrm{MCIR}}\right)$ and gated image reconstruction $\left(\hat{f}_{1}\right)$ will be

$$
E\left[\hat{\boldsymbol{f}}_{\mathrm{MCIR}}\right] \approx\left[\boldsymbol{G}_{0}^{\prime} \boldsymbol{G}_{0}+\eta \boldsymbol{C}_{0}^{\prime} \boldsymbol{C}_{0}\right]^{-1} \boldsymbol{G}_{0}^{\prime} \boldsymbol{G}_{0} \boldsymbol{f}_{1} \approx E\left[\hat{\boldsymbol{f}}_{1}\right]
$$

Note that gated image reconstruction uses a conventional $\kappa$ in [11]. 


\section{NOISE PROPERTIES}

In the usual case when $\boldsymbol{W}=\operatorname{Cov}\left\{\boldsymbol{y}_{\mathrm{c}}\right\}^{-1}$, the covariance of the MCIR estimator $\hat{f}_{\mathrm{MCIR}}$ is:

$$
\operatorname{Cov}\left\{\hat{\boldsymbol{f}}_{\mathrm{MCIR}}\right\}=\left[\boldsymbol{F}_{\mathrm{c}}+\eta \boldsymbol{R}\right]^{-1} \boldsymbol{F}_{\mathrm{c}}\left[\boldsymbol{F}_{\mathrm{c}}+\eta \boldsymbol{R}\right]^{-1} .
$$

To simplify, assume that we use the modified regularizer (10) and that the associated approximations hold. Then one can derive the following covariance approximation:

$$
\operatorname{Cov}\left\{\hat{\boldsymbol{f}}_{\mathrm{MCIR}}\right\} \approx D(1 / \underline{\kappa}) \boldsymbol{H}_{0}^{-1} \boldsymbol{F}_{0} \boldsymbol{H}_{0}^{-1} D(1 / \underline{\kappa}),
$$

where $\boldsymbol{H}_{0} \triangleq \boldsymbol{F}_{0}+\eta \boldsymbol{C}_{0}^{\prime} \boldsymbol{C}_{0}$.

The comparison of noise properties is only meaningful when the spatial resolutions are matched. If we assume that $\boldsymbol{G}_{m}=\boldsymbol{G}_{0}$, we can compare the noise covariances of MCIR and gated image reconstruction with matched spatial resolutions in the previous section. In this case, (14) becomes

$$
\operatorname{Cov}\left\{\hat{\boldsymbol{f}}_{\mathrm{MCIR}}\right\} \approx \frac{1}{M} D(1 / \underline{\kappa}) \Omega_{0} D(1 / \underline{\kappa})
$$

where

$$
\Omega_{0} \triangleq\left[\boldsymbol{G}_{0}^{\prime} \boldsymbol{G}_{0}+\eta \boldsymbol{C}_{0}^{\prime} \boldsymbol{C}_{0}\right]^{-1} \boldsymbol{G}_{0}^{\prime} \boldsymbol{G}_{0}\left[\boldsymbol{G}_{0}^{\prime} \boldsymbol{G}_{0}+\eta \boldsymbol{C}_{0}^{\prime} \boldsymbol{C}_{0}\right]^{-1} .
$$

We can compare (15) with the covariance of gated image reconstruction $\hat{f}_{1}$ with a conventional "static" choice for the regularization factors [11] given by:

$$
\kappa_{\mathrm{s} j} \triangleq \sqrt{\frac{\left[\boldsymbol{G}_{0}^{\prime} \boldsymbol{W}_{1} \mathbf{1}\right]_{j}}{\left[\boldsymbol{G}_{0}^{\prime} \mathbf{1}\right]_{j}}} .
$$

Then, one can show that the covariance of a single gate reconstruction is

$$
\operatorname{Cov}\left\{\hat{\boldsymbol{f}}_{1}\right\}=D\left(1 / \underline{\kappa}_{\mathrm{s}}\right) \Omega_{0} D\left(1 / \underline{\kappa}_{\mathrm{s}}\right) .
$$

The covariances of MCIR and gated image reconstruction in (15) and (17) depend on backprojected statistics. In addition, the covariance of MCIR also depends on the Jacobian determinant values, i.e., the local volume change.

\section{SIMULATION RESULTS}

We used 4 breathing phases of a 2D slice from the 4D XCAT phantom [14]. Ground truth motion was given by cubic Bspline nonrigid image registration based on true images. The original image has $128 \times 128$ image resolution respectively with $3.4 \mathrm{~mm}$ pixel size in Fig. 1. We placed 4 impulses in + locations for spatial resolution study. We forward projected these original images using the CTI 931 PET scanner geometry with 160 detector samples with $3.375 \mathrm{~mm}$ spacing 192 angular views, $3.375 \mathrm{~mm}$ strip width to model detector response and $10 \%$ random coincidences. We used $250 \mathrm{~K}$ mean true coincidences for each frame (1M total) and we ignored attenuation. We used 100 realizations for the covariance study.

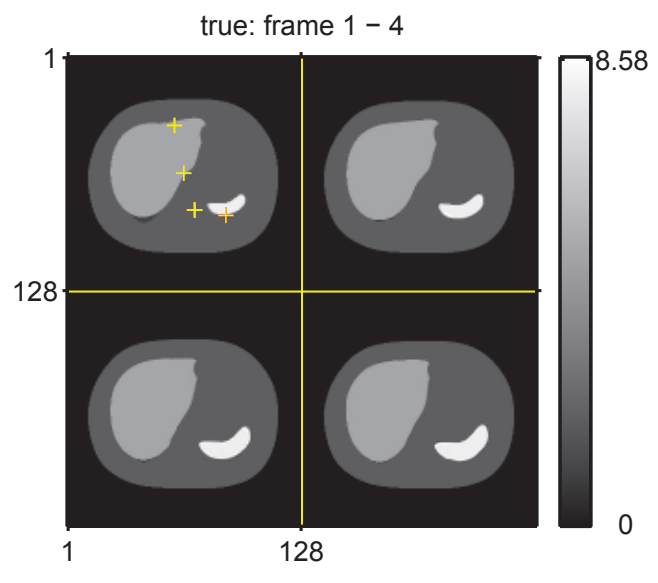

Fig. 1. 2D slices of 4D XCAT phantom at 4 different breathing phases.

We reconstructed $128 \times 128$ images from these 4 sinograms using the modified regularizer (10) to ensure consistent spatial resolution between methods. We compared the following methods: gated image reconstruction from a single sinogram with a conventional $\kappa$ (Gated), MCIR with a conventional $\kappa$ [11] (i.e., $\left[\boldsymbol{T}_{\mathrm{c}}^{\prime} \boldsymbol{G}_{\mathrm{d}}^{\prime} \mathbf{1}\right]_{j}$ in the denominator of (8)) and a proposed $\kappa$ in (8). We use a conjugate gradient method for all optimizations.
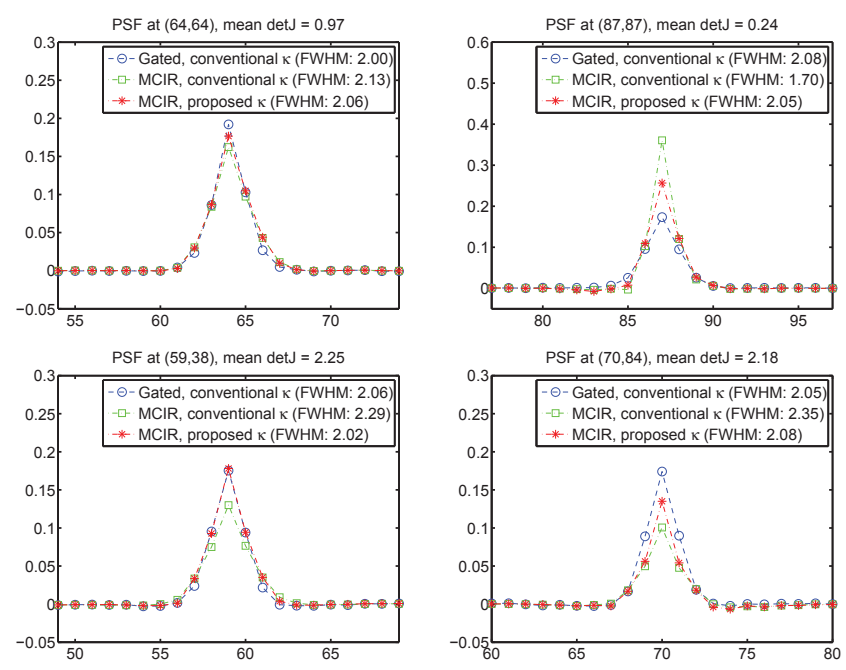

Fig. 2. Profile of local impulse responses from noiseless projections. Comparing to the conventional $\kappa$, the proposed $\kappa$ can match the spatial resolution with a gated image reconstruction case.

Reconstructed images from noiseless projections approximate the expectation of the estimated images [11]. Fig. 2 shows the profiles of local impulse responses (LIR) and fullwidth-half-maximum (FWHM) values at 4 different locations 
with different Jacobian determinant values. When the Jacobian determinant is close to 1 , the differences of LIR and FWHM with conventional and proposed $\kappa$ designs are small. However, for locations that experience large volume changes, proposed $\kappa(8)$ matches LIR and FWHM closer to the gated image reconstruction case.

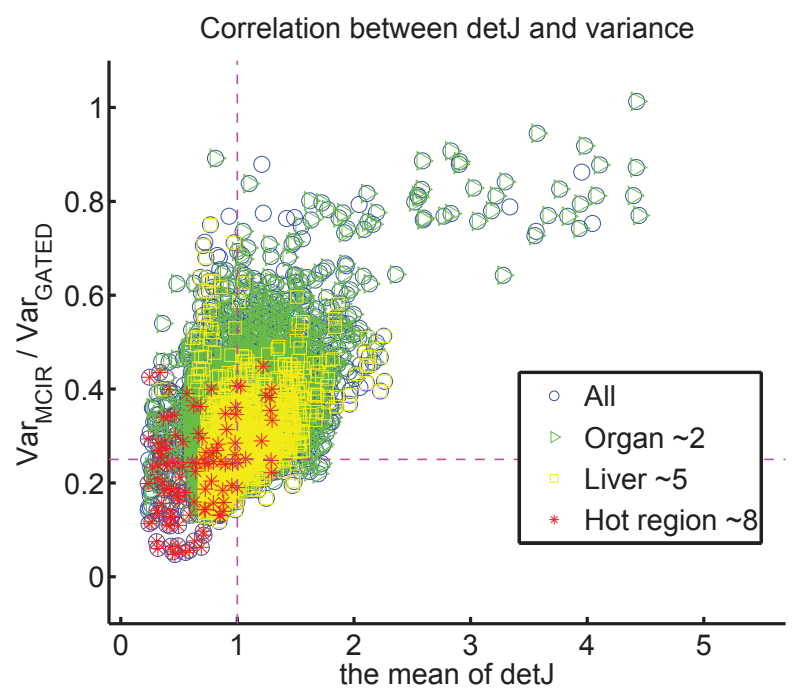

Fig. 3. Scatter plot of the mean of four Jacobian determinants (x-axis) vs. the ratio of variances of MCIR with a proposed $\kappa$ to Gated with a conventional $\kappa$ (y-axis).

Fig. 3 shows an empirical comparison of the relationship between the ratio of MCIR (with proposed $\kappa$ ) to Gated (with a conventional $\kappa$ ) and the mean of four Jacobian determinants of deformations. Since the variance of a Poisson model depends on the image intensity, we selectively plot the points in the different regions in different colors. This figure shows a correlation between variance and motion, as predicted.

\section{DISCUSSION}

This paper analyzed the spatial resolution and noise properties for MCIR methods. We proposed a regularization design method for MCIR that can provide approximately uniform spatial resolution regardless of object and motion. When $\boldsymbol{G}_{m}=\boldsymbol{G}_{0}$, one can approximately match the spatial resolution of MCIR with the resolution of gated image reconstruction (single frame). The analysis and results also showed that image variance depends on both measurement statistics and Jacobian determinant values for the proposed regularizer.

The theory in this paper can be applied to spatial-invariant $3 \mathrm{D}$ systems. We are currently extending the results to $3 \mathrm{D}$ MCIR. Extending the theory with fully 3D systems may require more careful investigation such as [9] and will be interesting.

\section{REFERENCES}

[1] F. Qiao, T. Pan, J. W. Clark, and O. R. Mawlawi, "A motionincorporated reconstruction method for gated PET studies," Phys. Med. Biol., vol. 51, no. 15, pp. 3769-84, Aug. 2006.

[2] T. Li, B. Thorndyke, E. Schreibmann, Y. Yang, and L. Xing, "Model-based image reconstruction for fourdimensional PET," Med. Phys., vol. 33, no. 5, pp. 1288-98, May 2006.

[3] F. Lamare, M. J. Ledesma Carbayo, T. Cresson, G. Kontaxakis, A. Santos, C. Cheze LeRest, A. J. Reader, and D. Visvikis, "List-mode-based reconstruction for respiratory motion correction in PET using non-rigid body transformations," Phys. Med. Biol., vol. 52, no. 17, pp. 5187-204, Sept. 2007.

[4] M. W. Jacobson and J. A. Fessler, "Joint estimation of image and deformation parameters in motion-corrected PET," in Proc. IEEE Nuc. Sci. Symp. Med. Im. Conf., 2003, vol. 5, pp. 3290-4.

[5] K. Taguchi, M. Zhang, E. C. Frey, J. Xu, W. Paul Segars, and B. M. W. Tsui, "Image-domain material decomposition using photon-counting CT," in Proc. SPIE 6510, Medical Imaging 2007: Phys. Med. Im., 2007, p. 651008.

[6] S. Y. Chun and J. A. Fessler, "Joint image reconstruction and nonrigid motion estimation with a simple penalty that encourages local invertibility," in Proc. SPIE 7258, Medical Imaging 2009: Phys. Med. Im., 2009, p. 72580 U.

[7] E. Asma, R. Manjeshwar, and K. Thielemans, "Theoretical comparison of motion correction techniques for PET image reconstruction," in Proc. IEEE Nuc. Sci. Symp. Med. Im. Conf., 2006, vol. 3, pp. 1762-7.

[8] J. A. Fessler, "Mean and variance of implicitly defined biased estimators (such as penalized maximum likelihood): Applications to tomography," IEEE Trans. Im. Proc., vol. 5, no. 3, pp. 493-506, Mar. 1996.

[9] J. Qi and R. M. Leahy, "Resolution and noise properties of MAP reconstruction for fully 3D PET," IEEE Trans. Med. Imag., vol. 19, no. 5, pp. 493-506, May 2000.

[10] E. Asma and R. M. Leahy, "Mean and covariance properties of dynamic PET reconstructions from list-mode data," IEEE Trans. Med. Imag., vol. 25, no. 1, pp. 42-54, Jan. 2006.

[11] J. A. Fessler and W. L. Rogers, "Spatial resolution properties of penalized-likelihood image reconstruction methods: Spaceinvariant tomographs," IEEE Trans. Im. Proc., vol. 5, no. 9, pp. 1346-58, Sept. 1996.

[12] S. Ahn and R. M. Leahy, "Analysis of resolution and noise properties of nonquadratically regularized image reconstruction methods for PET," IEEE Trans. Med. Imag., vol. 27, no. 3, pp. 413-24, Mar. 2008.

[13] J. A. Fessler, "Penalized weighted least-squares image reconstruction for positron emission tomography," IEEE Trans. Med. Imag., vol. 13, no. 2, pp. 290-300, June 1994.

[14] W. P. Segars, M. Mahesh, T. J. Beck, E. C. Frey, and B. M. W. Tsui, "Realistic CT simulation using the 4D XCAT phantom," Med. Phys., vol. 35, no. 8, pp. 3800-8, Aug. 2008. 